Revista Brasileira de Agricultura Irrigada v.14, n.4, p. 4106 - 4117, 2020

ISSN 1982-7679 (On-line)

Fortaleza, CE, INOVAGRI - http://www.inovagri.org.br

DOI: $10.7127 /$ rbai.v14n401166

Protocolo 1166.20 - 04/06/2020 Aprovado em 23/12/2020

\title{
EVAPOTRANSPIRAÇÃO DE REFERÊNCIA PELAS EQUAÇÕES DE HARGREAVES EM CLIMA TROPICAL
}

\author{
José Eduardo Pitelli Turco ${ }^{1}$
}

\begin{abstract}
RESUMO
A finalidade deste trabalho foi verificar a eficiência de três equações de Hargreaves, uma que emprega a radiação solar e a temperatura do ar e duas que utilizam a medição da temperatura do ar para estimativa da ETo, comparando-as com a equação de Penman-Monteith, nas condições climáticas do município de Jaboticabal, SP, bem como fazer a calibração local desta equação. Foram utilizados dados médios diários dos anos de 2010 a 2019, de radiação solar global, velocidade do vento, temperatura do ar e umidade relativa do ar, obtidos em uma estação meteorológica automática da Davis Instruments. A análise dos resultados foi realizada aplicando técnicas que verificam a integridade dos dados meteorológicos e utilizando metodologias de análise dos dados médios e desvios padrões da ETo. Foi realizada a calibração local das equações de Hargreaves por meio do ajuste do parâmetro empírico HE (expoente empírico de Hargreaves) das equações. As equações de Hargreaves são uma alternativa eficiente para estimar a ETo diária em clima subtropical, em que a disponibilidade de dados climáticos é limitada.
\end{abstract}

Palavras-chave: Penman-Monteith FAO-56, expoente empírico HE, temperatura do ar.

\section{REFERENCE EVAPOTRANSPIRATION BY THE HARGREAVES EQUATIONS IN SUBTROPICAL CLIMATE}

\begin{abstract}
This work aimed to verify the efficiency of three Hargreaves equations comparing them with the Penman-Monteith, in the climatic conditions of Jaboticabal, SP and calibrate this equation. For this purpose we collected data from Jan/01/2010 to Dec/31/2019 in an automated meteorological station installed at Department of Rural Engineering in FCAV / UNESP, Jaboticabal, SP Campus Experimental Area. The analysis of the results was carried out applying techniques that verify the integrity of the meteorological data as well using methodologies of analysis of the average data and standard deviations of the $\mathrm{ET}_{0}$. The local calibration of the Hargreaves equations was performed by

${ }^{1}$ Prof. Adjunto III, Dep. de Engenharia e Ciências Exatas, Universidade Estadual Paulista "Júlio de Mesquita Filho", Campus de Jaboticabal, CEP:14884-900, Jaboticabal, São Paulo, Brasil, (16) 32097278, E-mail: jose.turco@ unesp.br
\end{abstract}


adjusting the empirical parameter HE of the equation. The integrity of the weather station data was acceptable. The Hargreaves equation is an option to estimate $\mathrm{ET}_{0}$ values in subtropical climate in places where the climatic data availability is scarce.

Keywords: Penman-Monteith FAO-56, HE empirical exponent, air temperature.

\section{INTRODUÇÃO}

Para o manejo de irrigação precisamos saber quando e quanto irrigar. Para determinar quanto irrigar normalmente são utilizadas equações que estimam a evapotranspiração de referência (ETo), que é uma técnica indireta que conduz a estimativa das necessidades de água pelas plantas, uma vez utilizando o coeficiente de cultura (OLIVEIRA e TURCO, 2019).

A equação que estimativa a $\mathrm{ET}_{\mathrm{o}}$ recomendada pela FAO56 é de PenmanMonteith (PM). O processo de cálculo exige dados meteorológicos tais como: temperatura do ar, umidade relativa, radiação solar e velocidade do vento (LIMA JÚNIOR et al., 2016). Porém, há um número limitado de estações meteorológicas nos quais estes dados são medidos de modo eficiente. Portanto, a busca por métodos alternativos que demandem menos quantidade de variáveis meteorológicas para estimar a ETo têm sido uma solução viável para contornar esse problema (FERREIRA et al., 2018).

Os dados meteorológicos provenientes das estações automáticas devem apresentar qualidade para a estimativa da ETo. Avaliações da integridade e qualidade dos dados meteorológicos precisam ser realizadas antes dos dados serem utilizados em equações de estimativa da ETo (TURCO e CARLETO, 2017).

Antes de aplicar equações que estimam a $\mathrm{ET}_{\mathrm{o}}$ para determinado local, é necessário verificar o desempenho desta equação e, quando necessário, fazer calibrações a fim de minimizar erros de estimativa. Esse desempenho tem sido analisado com a comparação das equações em estudo em relação a equação de Penman-Monteith FAO-
56 (BORGES JÚNIOR et al., 2012).

Uma equação alternativa que vem sendo utilizada em vários estudos (RAZIEI e PEREIRA.; 2013; HEYDARI et al., 2014; MEHDIZADEH et al., 2016) é a de Hargreaves e Samani (1985).

Entre as equações encontradas na literatura a que se destaca é a de Hargreaves (1994). Estudos mostram que essa equação ajustada pode estimar com precisão a ETo diária (BERTI et al., 2014; FENG et al., 2017).

O desempenho da equação de HARGREAVES (1994) no Vale de Bekaa Líbano, foi avaliado por Bachour et al. (2013) por meio da comparação com o método de Penman-Monteith FAO-56.

A equação de Hargreaves (1994) superestima a $\mathrm{ET}_{0}$ em locais de clima úmido, conforme observado por Cervantes-Osornio et al. (2013).

Mendicino e Senatone (2013) fizeram a calibração local e regional da equação de Hargreaves (1994) para o sul da Itália, comparando com a equação de PenmanMonteith FAO-56, utilizando-se dados do período de 1994 a 2011 de 34 estações costeiras e 103 no interior. A melhoria no desempenho da equação regional em relação a original foi de $22 \%$.

Com este trabalho o objetivo foi verificar a eficiência de três equações de Hargreaves comparando-as com a equação de Penman-Monteith, para verificar a possibilidade de estimar a evapotranspiração de referência para as condições climáticas do município de Jaboticabal - SP. Fazer a calibração local das equações, baseadas na temperatura do ar, por meio do ajuste do parâmetro empírico HE (expoente empírico de Hargreaves). 


\section{TROPICAL}

\section{MATERIAIS E MÉTODOS}

A pesquisa foi desenvolvida na área experimental do Departamento de Engenharia Rural da FCAV/UNESP, Campus de Jaboticabal, SP, situada em torno das coordenadas geográficas $21^{\circ} 15^{\prime} 22^{\prime \prime}$ S e

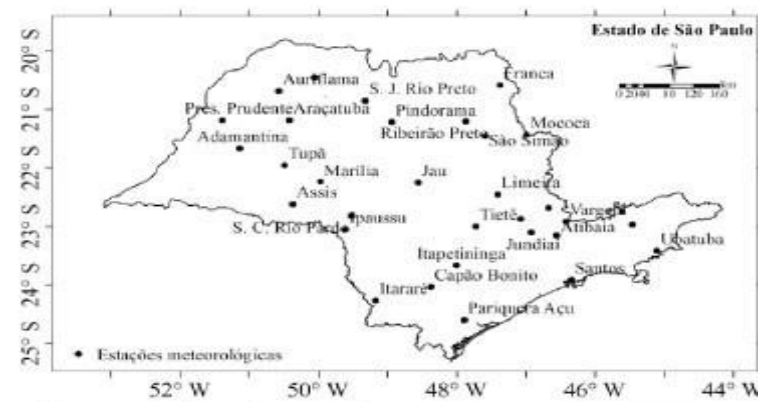

latitude $48^{\circ} 18^{\prime} 58^{\prime \prime} \mathrm{W}$ e altitude de $575 \mathrm{~m}$, na cidade de Jaboticabal, SP. Jaboticabal situa-se na região agropecuária noroeste do estado de São Paulo, na região Administrativa e de Governo de Ribeirão Preto, composta por outros 25 municípios conforme mapa a seguir.

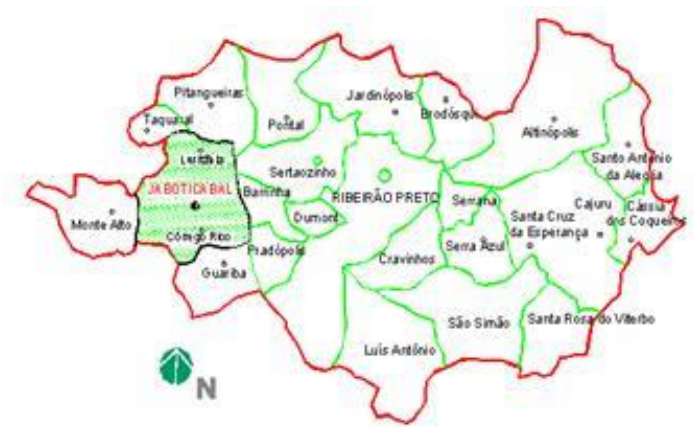

Figura 1. Mapa do Estado de São Paulo. Fonte: Camparotto et al. (2013), Região Administrativa e de Governo de Ribeirão Preto e Jaboticabal - SP. Fonte: https://www.achetudoeregiao.com.br/sp/jaboticabal/localizacao.htm

O clima de acordo com a classificação de Köppen, é do tipo Cwa (subtropical). $\mathrm{Na}$ área experimental foi plantada grama batatais (Paspalum notatum Flügge), cobrindo totalmente o solo.

Os dados meteorológicos foram fornecidos pelo Laboratório de Instrumentação, Automação e Processamento do Departamento de Engenharia Rural da FCAV/UNESP-Câmpus de Jaboticabal. Esses dados foram obtidos em uma estação meteorológica automática da marca Davis Instruments instalada em área experimental do Departamento de Engenharia Rural (DER).

A estação possui os seguintes sensores: temperatura e umidade relativa do ar, modelo 7859; velocidade do vento, modelo 7911; radiação solar global, modelo 6450; todos da marca Davis Instruments. Os sensores de radiação solar, temperatura do ar e umidade relativa do ar foram colocados a 1,5 metros acima da superfície gramada e os sensores de velocidade do vento, a 2 metros da superfície gramada.

Foram utilizados dados de temperatura do ar, umidade relativa do ar, velocidade do vento e radiação solar global, do período de 01/01/2010 a 31/12/2019.

A integridade dos dados da estação meteorológica automática da marca Davis
Instruments foi avaliada pelas técnicas descritas por Allen (1996).

Os dados da estação meteorológica automática da marca Davis Instruments foram comparados aos da estação meteorológica automática da marca Campbell Scientific (Estação de Referência), instalada ao seu lado. Metodologia semelhante foi utilizada e descrita por Turco e Carleto (2017).

A ETo foi estimada em escala média diária, para os anos de 2010 a 2019, pelas equações de Hargreaves (1976), Hargreaves e Samani (1985), Hargreaves (1994) e PenmanMonteith FAO56 (ALLEN et al., 2006), considerando esse último como padrão para a comparação dos demais métodos. A seguir são apresentadas as equações utilizadas neste estudo.

\section{Equação de Hargreaves (1976)}

$$
\mathrm{EToH}=0,0075 \mathrm{Q} \mathrm{T}
$$

Em que: EToH - evapotranspiração de referência, em gramado, $\mathrm{mm} \mathrm{dia}^{-1} ; \mathrm{T}$ temperatura média do ar, ${ }^{\circ} \mathrm{F} ; \mathrm{Q}$ - radiação solar medida, cal cm $\mathrm{cmia}^{-1}$.

Pode-se escrever a eq. (1) na seguinte forma: 
Turco J. E. P.

$$
E T o H=0,0075 \frac{Q}{59} T
$$

\section{Equação de Hargreaves e Samani (1985)}

EToHS $=0,0023$ Qo $(\text { Tmáx }- \text { Tmín })^{\mathrm{HE}}(\mathrm{T}+17,8)$

Em que: EToHS - evapotranspiração de referência, grama, $\mathrm{mm} \mathrm{d}^{-1}$; Tmáx temperatura máxima, ${ }^{\circ} \mathrm{C}$; Tmín - temperatura

\section{Equação de Hargreaves (1994)}

$$
\mathrm{EToHG}=0,0023 \mathrm{RA}\left(\mathrm{T}^{\circ} \mathrm{C}+17,8\right) \mathrm{TD}^{\mathrm{HE}}
$$

Em que: EToHG - evapotranspiração de referência, em gramado, $\mathrm{mm} \mathrm{dia}{ }^{-1}$; RA radiação solar extraterrestre $\left(\mathrm{mm} \mathrm{dia}^{-1}\right)$; $\mathrm{T}^{\circ} \mathrm{C}$ $($ Tmax + Tmin)/2 (temperatura máxima diária do ar mais a temperatura mínima diária do ar,

Os valores diários da radiação solar extraterrestre para equação Hargreaves e Samani (1985) e Hargreaves (1994) foram

\section{Equação do Penman-Monteith (PM)}

$$
\operatorname{EToPM}=\frac{0,409 \Delta(R n-G)+\gamma\left(\frac{900}{T+273}\right) v\left(e_{s}-e\right)}{\Delta+\gamma\left(1+0,34_{v}\right)}
$$

Em que: EToPM - evapotranspiração de referência, em gramado, $\mathrm{mm} \mathrm{d}^{-1} ; \mathrm{Rn}$ - radiação líquida, $\mathrm{MJ} \mathrm{m}^{-2} \mathrm{~d}^{-1} ; \mathrm{G}$ - fluxo de calor no solo, $\mathrm{MJ} \mathrm{m}{ }^{-2} \mathrm{~d}^{-1} ; \mathrm{T}$ - temperatura média do ar, ${ }^{\circ} \mathrm{C} ; \mathrm{V}$ - velocidade média do vento a $2 \mathrm{~m}$ de altura,

Para a avaliação dos dados médios diários da ETo foi utilizada análise de regressão e considerando o modelo linear $(y=$ $\mathrm{a} x+\mathrm{b}$ ), na qual a variável dependente foram as estimativas da ETo pelos métodos estudados e o método de Penman-Monteith FAO-56 a variável independente. Para atingir o objetivo desse trabalho foi aplicada a técnica de Turco et al. (1994) nos métodos estudados para determinação dos desvios padrões diários da ETo.
Em que: 1/59 - fator de conversão de cal $\mathrm{cm}^{-2}$ a mm de evaporação equivalente.

mínima, ${ }^{\circ} \mathrm{C} ; \mathrm{T}$ - temperatura média diária, ${ }^{\circ} \mathrm{C}$; Qo - radiação solar extraterrestre $\left(\mathrm{mm} \mathrm{dia}^{-1}\right)$; $\mathrm{HE}$ - expoente empírico $(0,5)$.

em ${ }^{\circ} \mathrm{C}$, dividido por dois); TD - Tmax - Tmin (temperatura máxima diária do ar menos a temperatura mínima diária do ar, em $\left.{ }^{\circ} \mathrm{C}\right) ; \mathrm{HE}$ - expoente empírico $(0,5)$.

obtidos em função da latitude local, a partir dos dados interpolados de Napier Shaw, citado por Ramos et al. (1989).

$\mathrm{m} \mathrm{s}^{-1} ;\left(\mathrm{e}_{\mathrm{s}}\right.$ - e) - déficit de pressão de vapor, $\mathrm{kPa} ; \Delta$ - curva de pressão de vapor, $\mathrm{kPa}^{\circ} \mathrm{C}^{-1} ; \gamma$ - constante psicrométrica, $\mathrm{kPa} \quad{ }^{\circ} \mathrm{C}^{-1} ; 900$ - fator de conversão.

Para a análise dos resultados, considerando os desvios-padrão (erros) da $\mathrm{ET}_{0}$, foram utilizadas as metodologias de Vuolo (1996), da transferência do erro da variável independente para a variável dependente, o método dos mínimos quadrados e a avaliação da qualidade do ajuste.

Um critério de avaliação da qualidade do ajuste é essencialmente um método para se determinar se a curva ajustada foi boa em relação aos pontos experimentais. $\mathrm{O}$ critério 
utilizado para avaliação da qualidade de ajuste foi o Teste de $X^{2}$ reduzido, sendo a quantidade $\mathrm{X}^{2}$ reduzido definida como:

$$
X_{\text {red }}^{2}=\frac{X^{2}}{v}
$$

Em que: $X^{2}$ - é a soma dos quadrados das distâncias dos pontos experimentais à curva ajustada, e $v$ - é o número de graus de liberdade do ajuste.

Como $\mathrm{n}$ é o número de pontos experimentais e p o número de parâmetros ajustados $v=(\mathrm{n}-\mathrm{p})$.
Após a obtenção dos $X_{r e d}^{2}$, a avaliação da qualidade de ajuste foi feita por meio da metodologia de Vuolo (1996) que relaciona $v$ com $X_{\text {red }}^{2}$ permitindo obter, para cada $v$, um intervalo de confiança $\mathrm{P}=98 \%$, para os valores de $X_{\text {red }}^{2}$.

$\mathrm{Na}$ metodologia de Vuolo (1996), os valores de $v$ vão até 160 e, entretanto, em nosso trabalho, $v=363$, que corresponde aos 365 dias do ano utilizados no estudo e os dois parâmetros ajustados (EToH e EToHGL). Para EToHS $v=362$, que corresponde aos 365 dias do ano utilizados no estudo e três parâmetros ajustados.

Foram feitos os seguintes procedimentos para determinar o $\chi_{\text {red }}^{2}(1 \%$ e 99\%):

PROGRAMA MICROSOFT EXCEL / FUNÇÃO ESTATÍSTICA / INV.QUI para $v=363 \mathrm{e}$ $1 \%$ é obtido $X^{2}=428,61$; portanto $\quad X_{r e d}^{2}=\frac{X^{2}}{v}=1,181$ e INV.QUI para $v=362$ e $1 \%$ é obtido $\mathrm{X}^{2}=427,53$; portanto $\quad X_{\text {red }}^{2}=\frac{X^{2}}{v}=1,181$

PROGRAMA MICROSOFT EXCEL / FUNÇÃO ESTATÍSTICA / INV.QUI para $v=363 \mathrm{e}$ $99 \%$ é obtido $X^{2}=303,27$; portanto $X_{r e d}^{2}=\frac{X^{2}}{v}=0,835$ e INV.QUI para $v=362$ e $99 \%$ é obtido $\mathrm{X}^{2}=302,30 ;$ portanto $\quad X_{\text {red }}^{2}=\frac{X^{2}}{v}=0,835$

Pode-se afirmar com $98 \%$ de confiança que: $0,835<\chi_{\text {red }}^{2}<1,181$.

Foi realizada a calibração local das equações de Hargreaves (EToHSL e EToHGL) por meio do ajuste do expoente empírico HE das equações.

Para calibração local das equações foi utilizada a metodologia utilizada por Trajkovic (2007), utilizando os dados meteorológicos dos anos ímpares (2011, 2013, 2015, 2017 e 2019). Para validação da equação foi utilizado os dados meteorológicos dos anos pares (2010, 2012, 2014, 2016 e 2018). Esta metodologia reduz o efeito bias que pode ocorrer de um ano para o outro.

\section{RESULTADOS E DISCUSSÃO}

A partir das técnicas descritas por Allen (1996) foi realizada a análise da integridade dos dados. Os resultados seguintes referem-se ao ano de 2019, pois os resultados dos outros anos $(2010,2011,2012,2013,2014,2015$, 2016, 2017 e 2018) apresentaram semelhança.

Na Figura 2 de acordo com a observação da radiação solar medida pela Davis pode-se

identificar que a mesma é de qualidade, pois apresenta valores iguais ou inferiores ao apresentado pela radiação solar que foi estimada pelas equações de Allen (1996), corroborando com resultados obtidos por Turco e Barbosa (2008), Oliveira (2014) e Turco e Carleto (2017). 
Turco J. E. P.

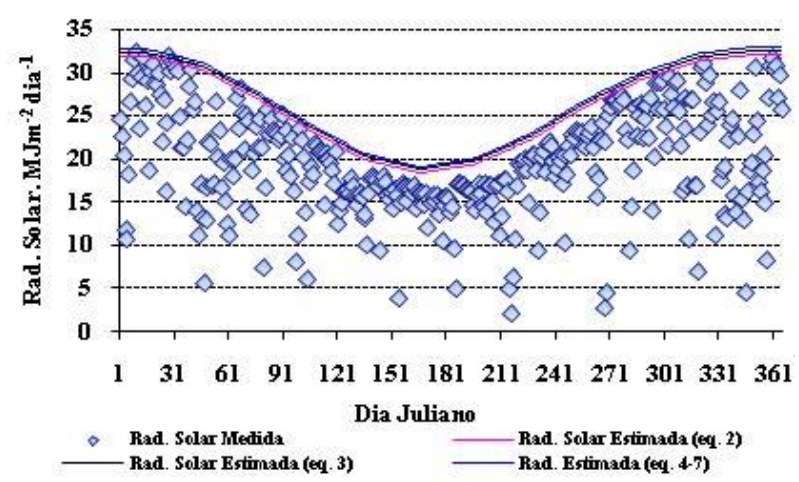

Figura 2. Radiação solar medida pela estação meteorológica automática da DAVIS e Radiação Solar estimada por três equações (eq.2, eq.3 e eq.4-7) das técnicas descritas por Allen (1996).

Pode-se verificar na Figura 3 os registros de velocidade do vento das estações meteorológicas, sendo comparados os valores de acordo com a técnica de dupla massa. Foi observado diferença dos valores averiguados na forma acumulativa dos anemômetros e isto se deve ao fato do offset aplicado na programação das estações.

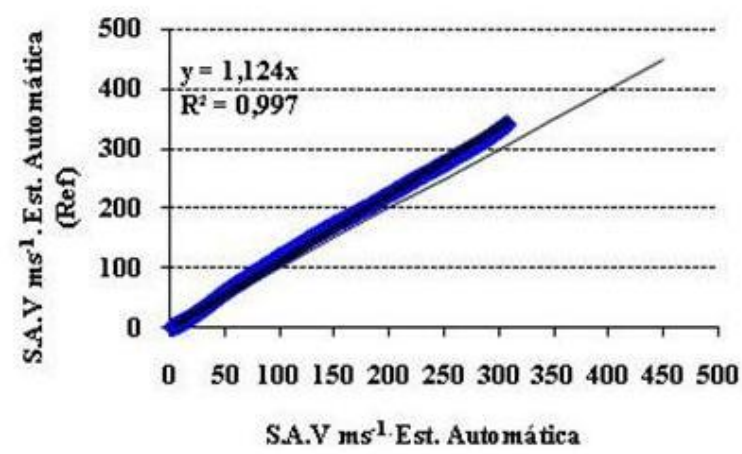

Figura 3. Soma acumulativa da Velocidade do Vento das estações meteorológicas de Referência e da Davis

A estação Davis e a de referência utilizam respectivamente um offset $0,0 \mathrm{~m} \mathrm{~s}^{-1} \mathrm{e}$ 0,2 $\mathrm{m} \mathrm{s}^{-1}$, Turco e Carleto (2017), em suas pesquisas alcançaram resultados semelhantes quando verificaram os dados obtidos pela Davis em comparação com a estação de referência.

Com relação a umidade relativa esta apresentou valores semelhantes a da estação de referência mostrando que a mesma está com desempenho adequado (Figura 4).

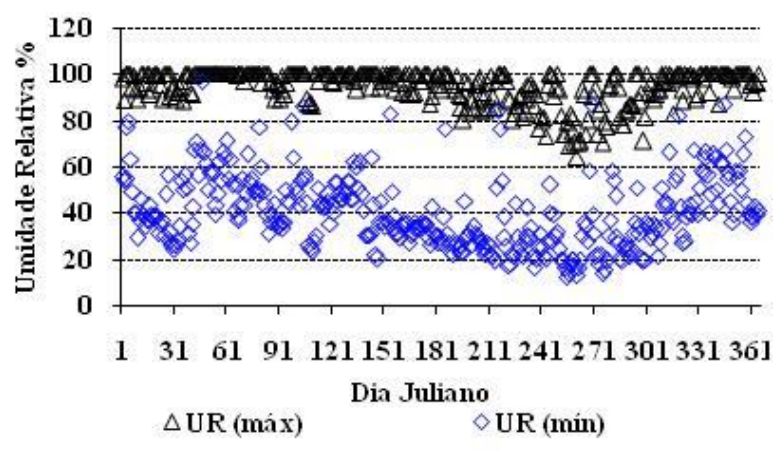

Figura 4. Valores diários da Umidade Relativa Máxima e Umidade Relativa Mínima da estação meteorológica da Davis.

Os valores obtidos referentes aos parâmetros temperaturas máxima e mínima apresentaram gráficos com inclinação de reta formando um ângulo de $45^{\circ}$ representados nas Figuras 5 e 6, indicam um alto grau de correlação entre os dados, pois os pontos apresentam-se bem próximos a reta fazendo com que o $\quad \mathrm{R}^{2}$ seja superior a 0,7 demonstrando assim, boa qualidade dos dados de acordo com Oliveira (2014).

A Tabela 1 mostra $\mathrm{o}$ resultado da análise de regressão dos métodos de Hargreaves (1976), Hargreaves e Samani (1985), Hargreaves (1994), para os anos de 2010 a 2019. 


\section{TROPICAL}
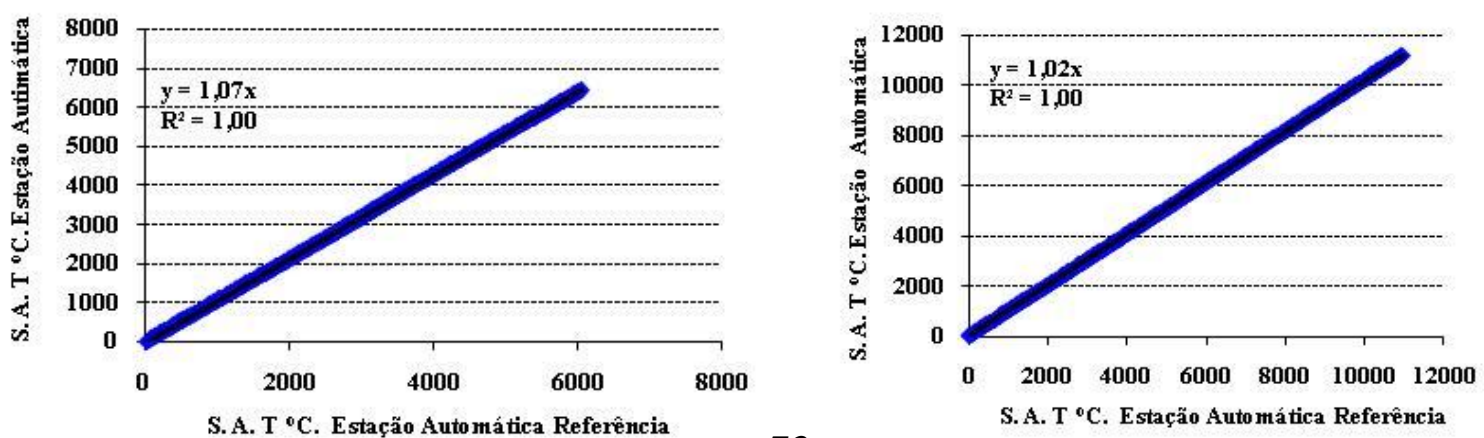

73

Figura 5. Soma acumulativa da Temperatura Mínima das estações meteorológicas de referência e da Davis (A). Soma acumulativa da Temperatura Máxima das estações meteorológicas de referência e da Davis (B).

Foi considerando o modelo linear $(\mathrm{y}=$ $\mathrm{ax}+\mathrm{b}$ ), na qual a variável dependente foram as estimativas da ETo pelos métodos estudados e o método de Penman-Monteith FAO-56 a variável independente.

Tabela 1. Valores do coeficiente de determinação $\left(\mathrm{R}^{2}\right)$ e equação $(\mathrm{y}=\mathrm{a} \mathrm{x}+\mathrm{b})$ para os métodos estudados.

\begin{tabular}{ccccccc}
\hline \multirow{2}{*}{ ANO } & \multicolumn{2}{c}{ Hargreaves (1976) } & \multicolumn{2}{c}{ Hargreaves e Samani (1985) } & \multicolumn{2}{c}{ Hargreaves (1994) } \\
\cline { 2 - 7 } & Equação & $\mathrm{R}^{2}$ & Equação & $\mathrm{R}^{2}$ & Equação & $\mathrm{R}^{2}$ \\
\hline 2010 & $\mathrm{y}=0,809 \mathrm{x}+0,611$ & 0,889 & $\mathrm{y}=0,921 \mathrm{x}-0,077$ & 0,794 & $\mathrm{y}=0,929 \mathrm{x}-0,166$ & 0,780 \\
2011 & $\mathrm{y}=0,809 \mathrm{x}+0,461$ & 0,931 & $\mathrm{y}=0,916 \mathrm{x}-0,211$ & 0,789 & $\mathrm{y}=0,93 \mathrm{x}-0,328$ & 0,787 \\
2012 & $\mathrm{y}=0,823 \mathrm{x}+0,414$ & 0,947 & $\mathrm{y}=0,846 \mathrm{x}+0,056$ & 0,817 & $\mathrm{y}=0,85 \mathrm{x}-0,014$ & 0,811 \\
2013 & $\mathrm{y}=0,826 \mathrm{x}+0,290$ & 0,97 & $\mathrm{y}=0,884 \mathrm{x}-0,186$ & 0,833 & $\mathrm{y}=0,886 \mathrm{x}-0,256$ & 0,828 \\
2014 & $\mathrm{y}=0,836 \mathrm{x}+0,377$ & 0,93 & $\mathrm{y}=0,888 \mathrm{x}-0,171$ & 0,804 & $\mathrm{y}=0,909 \mathrm{x}-0,311$ & 0,804 \\
2015 & $\mathrm{y}=0,831 \mathrm{x}+0,177$ & 0,882 & $\mathrm{y}=0,778 \mathrm{x}+0,314$ & 0,775 & $\mathrm{y}=0,771 \mathrm{x}+0,3$ & 0,769 \\
2016 & $\mathrm{y}=0,882 \mathrm{x}+0,265$ & 0,96 & $\mathrm{y}=0,933 \mathrm{x}-0,309$ & 0,725 & $\mathrm{y}=0,911 \mathrm{x}-0,289$ & 0,709 \\
2017 & $\mathrm{y}=0,851 \mathrm{x}+0,438$ & 0,918 & $\mathrm{y}=0,99 \mathrm{x}-0,062$ & 0,865 & $\mathrm{y}=0,995 \mathrm{x}-0,133$ & 0,858 \\
2018 & $\mathrm{y}=0,798 \mathrm{x}+0,597$ & 0,96 & $\mathrm{y}=1,033 \mathrm{x}-0,451$ & 0,802 & $\mathrm{y}=1,03 \mathrm{x}-0,496$ & 0,79 \\
2019 & $\mathrm{y}=0,82 \mathrm{x}+0,488$ & 0,938 & $\mathrm{y}=1,03 \mathrm{x}-0,411$ & 0,853 & $\mathrm{y}=1038 \mathrm{x}-0,513$ & 0,849 \\
\hline
\end{tabular}

A Tabela 1 mostra que, os valores obtidos da análise dos métodos de Hargreaves apresentaram bons resultados para o local de estudo, por apresentarem coeficientes de determinação maiores que 0,71. Resultado semelhante foi obtido por Silva et al.(2014), que a equação de Hargreaves (1976) foi o que se mostrou mais coerente e confiável para a evapotranspiração diária e também por Lacerda e Turco (2015), que verificaram que a análise de regressão indicou ajuste mais eficiente para estimativa da $\mathrm{ET}_{0}$ para a equação de Hargreaves (1976), comparando com a equação de Penman-Monteith.

$\mathrm{Na}$ Figura 7, pode-se observar a correlação dos valores diários da evapotranspiração de referência obtidos por meio dos métodos de Hargreaves, média dos anos de 2010 a 2019, em relaçãoao método de Penman-Monteith FAO-56, para região do município de Jaboticabal $-\mathrm{SP}$. 

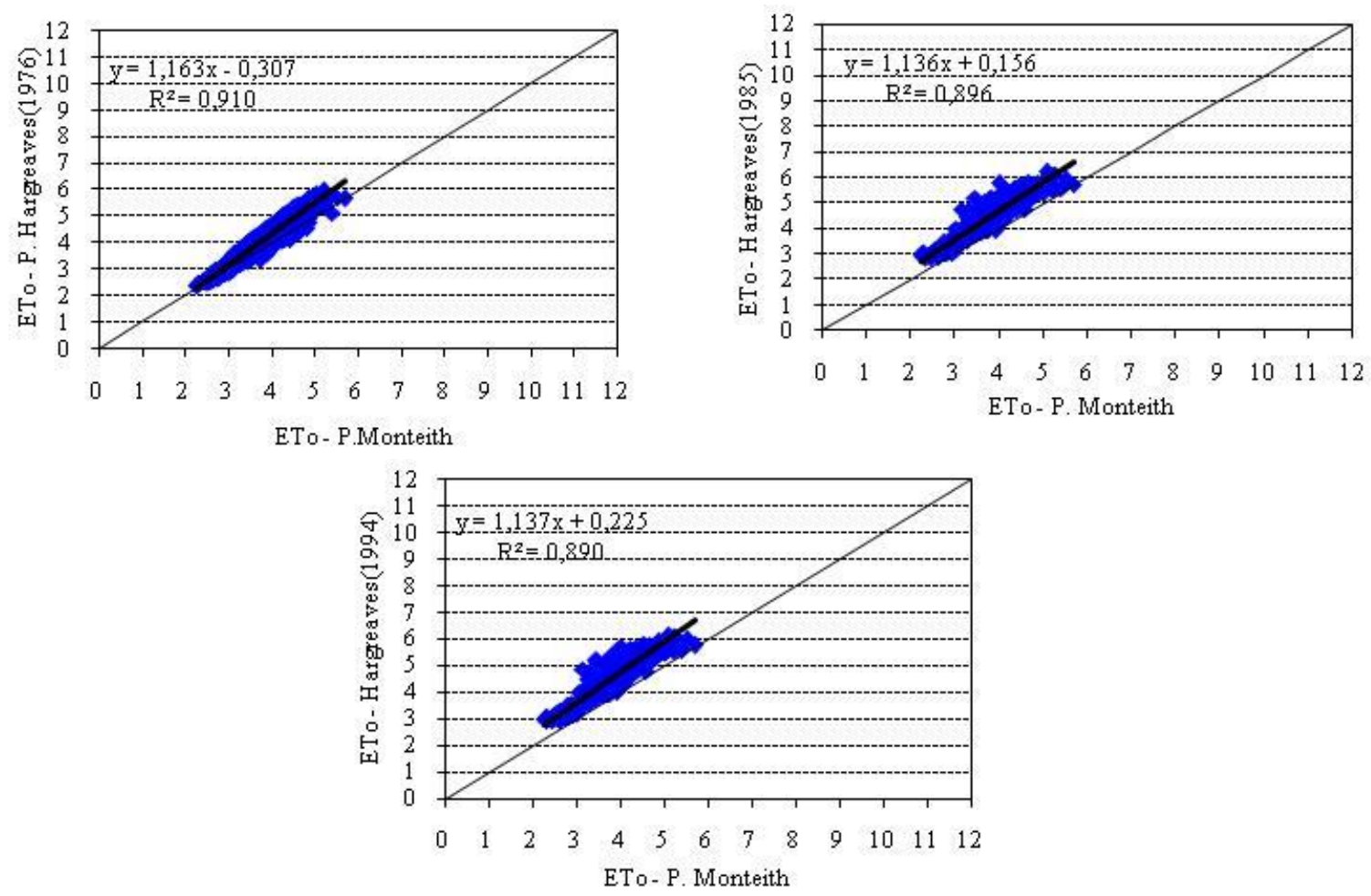

Figura 7. Correlação entre a os valores diários da ETo, obtidos com os métodos estudados, em relação ao Penman Monteith FAO-56.

Essas comparações fornecem a base para avaliar os valores diários da ETo, obtidos pelos métodos de Hargreaves em relação aos obtidos pelo método de Penman-Monteith FAO-56. Se os valores diários da ETo obtidos pelo método de Penman-Monteith FAO-56 forem semelhantes aos obtidos pelos métodos de Hargreaves a linha de regressão deveria sobrepor-se à reta $\mathrm{y}=\mathrm{x}$, e os pares de pontos deveriam estar próximos à linha de regressão.

Quando a linha de regressão estiver afastada da reta $\mathrm{y}=\mathrm{x}$, e os pares de pontos estiverem próximos à linha de regressão, isso significa que os valores obtidos pelos métodos de Hargreaves apresentam uma diferença aceitável em relação aos obtidos pelo método de Penman-Monteith FAO-56, ou seja, a equação pode ser utilizada com precisão para fazer a correção dos valores obtidos pelos métodos Hargreaves em relação aos obtidos pelo método de Penman-Monteith FAO-56 .

Observa-se na Figura 7 que a linha de regressão obtida pelos valores da ETo obtidos pelos métodos de Hargreaves, média dos anos de 2010 a 2019, em relação aos obtidos pelo método de Penman-Monteith FAO-56 superestimaram a ETo.

Referente também a Figura 7, percebe-se que os pares de pontos não estão dispersos ao redor da linha de regressão, indicando uma adequada correlação, podendo-se observar isto também por meio do coeficiente de determinação $\mathrm{R}^{2}$.

Resultados semelhantes foram obtidos por Bachour et al. (2013), no Vale de Bekaa Líbano, onde o método Hargreaves (1994) superestimou a ETo em relação ao método de Penman-Monteith FAO-56.

Por meio da metodologia de Vuolo (1996), as retas $y=a x+b$ foram ajustadas aos pontos experimentais, obtidos diariamente pelos métodos estudados, nos anos de 2010 a 2019, em relação ao método de PenmanMonteith FAO-56 e respectivas incertezas.

No ajuste das retas para os métodos de Hargreaves foram obtidos os valores de $\chi_{\text {red }}^{2}$ apresentados na Tabela 2. De acordo com Vuolo (1996), os valores de qui-quadrado 
reduzido $\left(\chi_{\text {red }}^{2}\right)$ estão dentro da faixa de

portanto considerados bons ajuste.

valores aceitáveis $\left(0,835<\quad \chi_{\text {red }}^{2}<1,181\right)$,

Tabela 2. Valores do qui-quadrado reduzido $\chi_{\text {red }}^{2}$ para os métodos estudados.

\begin{tabular}{cccc}
\hline \multirow{2}{*}{ ANO } & Hargreaves (1976) & $\begin{array}{c}\text { Hargreaves e Samani } \\
(1985)\end{array}$ & Hargreaves (1994) \\
\cline { 2 - 4 } & $\chi_{\text {red }}^{2}$ & $\chi_{\text {red }}^{2}$ & $\chi_{\text {red }}^{2}$ \\
\hline 2010 & 1,128 & 1,176 & 1,177 \\
2011 & 1,009 & 1,057 & 1,057 \\
2012 & 0,915 & 0,865 & 0,865 \\
2013 & 1,04 & 0,989 & 0,989 \\
2014 & 0,984 & 0,933 & 0,933 \\
2015 & 0,993 & 1,044 & 1,044 \\
2016 & 0,984 & 1,033 & 1,033 \\
2017 & 1,036 & 0,983 & 0,984 \\
2018 & 0,895 & 0,84 & 0,84 \\
2019 & 1,028 & 1,074 & 1,074 \\
\hline
\end{tabular}

De acordo com a estatística há $98 \%$ de confiança, que os métodos estudados e o método de Penman-Monteith não são distintos, assim podem ser comparados.

O Resultado obtido foi similar ao de Lacerda e Turco (2015), que compararam o método de estimativa da ETo de Hargreaves (1976) com o método de Penman-Monteith FAO-56, para Uberlandia - MG, utilizando a metodologia de análise de desvios padrões

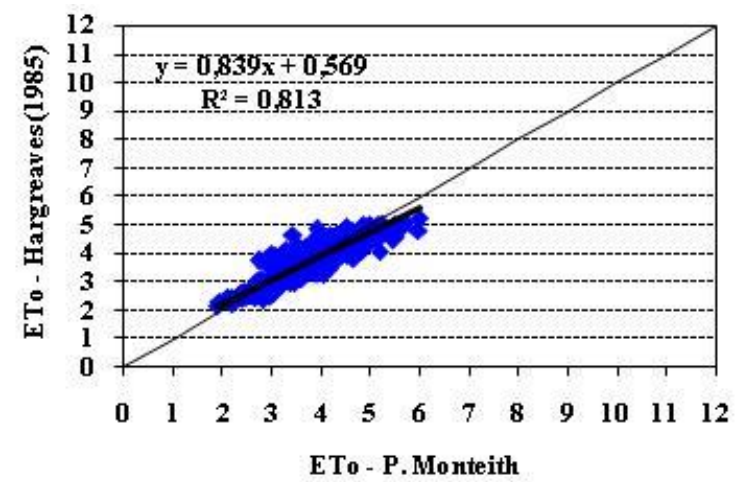

Diários da ETo.

$\mathrm{Na}$ Figura 8 pode-se observar a correlação dos valores diários da evapotranspiração de referência obtidos por meio das equações de Hargreaves com calibração local (EToHSL e EToHGL), durante os anos de 2010, 2012, 2014, 2016 e 2018 em relação ao método de PenmanMonteith FAO-56, para região do município de Jaboticabal - SP.

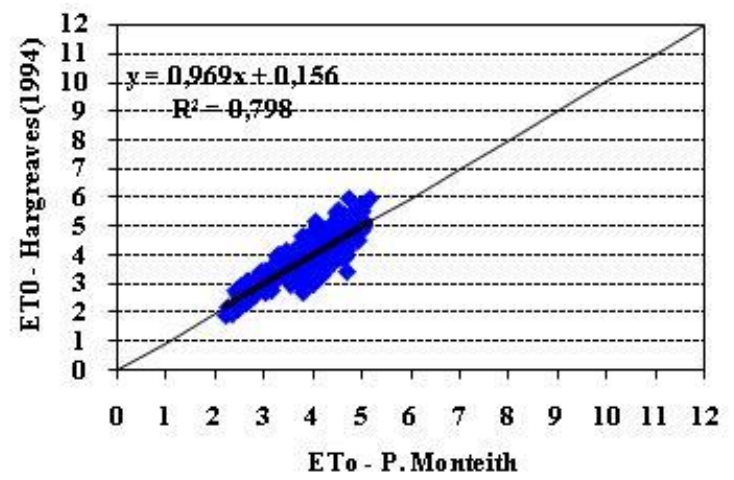

Figura 8. Correlação entre a os valores diários da ETo, obtidos com os métodos estudados com calibração local, em relação ao Penman-Monteith FAO-56 (Anos: 2010, 2012, 2016 e 2018).

Observa-se na Figura 8 que a linha de regressão sobrepõe a reta $\mathrm{y}=\mathrm{x}$, e os pares de pontos estão próximos à linha de regressão, indicando uma adequada correlação, podendose observar isto também por meio do coeficiente de determinação $\mathrm{R}^{2}$. Por meio da calibração local das equações de Hargreaves (EToHSL e EToHGL) foram obtidos os valores de $\operatorname{HE}(0,43$ e 0,424). Os valores obtidos corroboram com o obtido por Trajkovic (2007). 


\section{CONCLUSÕES}

A integridade dos dados da estação meteorológica da Davis foi aceitável.

As equações de Hargreaves apresentaram um bom ajuste para estimativa da ETo, considerando a análise de regressão e a metodologia que verifica a influência do desvio padrão diário da $\mathrm{ET}_{0}$.

As equações de Hargreaves com calibração local é uma alternativa para estimar a ETo diária em locais em que a disponibilidade de dados meteorológicos é limitada.

\section{REFERÊNCIAS}

ALLEN, R. G. Assessing integrity of weather data for reference evapotranspiration estimation. Journal of Irrigation and Drainage Engineering, v. 122, p. 97-106, 1996. https://doi.org/10.1061/(ASCE)07339437(1996)122:2(97)

ALLEN, R. G.; PEREIRA, L. S.; RAES, D.; SMITH, M. Evapotranspiración del cultivo: guías para la determinación de los requerimientos de agua de los cultivos. Roma: FAO, 2006. 298 p. (Estudio Riego e Drenaje, $56)$.

BACHOUR, R.; WALKER, W.R.; TORRESRUA, A.F.; MCKEE, M. Assessment of reference evapotranspiration by the Hargreaves method in the Bekaa Valley, Lebanon. Journal of Irrigation and Drainage Engineering, North Carolina, v. 139, n. 11, p. 933-938, 2013.

BERTI, A.; TARDIVO, G.; CHIAUDANI, A.; RECH, F.; BORIN, N. Assessing reference evapotranspiration by the Hargreaves method in north-eastern Italy. Agricultural Water Management. v.140, p.20-25, 2014. https://doi.org/10.1016/j.agwat.2014.03.015
BORGES JÚNIOR, J.C.F.; ANJOS, R.J.; SILVA, T.J.A.; LIMA, J.R.S.; ANDRADE, C.L.T. Métodos de estimativa da evapotranspiração de referência diária para a microrregião de Garanhuns, PE. Revista Brasileira de Engenharia Agrícola e Ambiental, Campina Grande, v.16, n.4, p.380-390, 2012.

CAMPAROTTO, L. B.; BRAIN, G. S.; GIAROLLA, A.; ADAMI, M.; CAMARGO, M.B.P.de.Validação de dados termopluviométricos obtidos via sensoriamento remoto para o Estado de São Paulo.. Revista Brasileira de Engenharia Agrícola e Ambiental, Campina Grande, v. 17, n.6, p. 665-671, 2013.

CERVANTES-OSORNIO, R.; ARTEAGARAMÍRES, R.; VÁZQUEZ-PEÑA, M.A.; OJEDA-BUSTAMANTE, W.; QUEVEDONOLASCO, A. Modelos Hargreaves Priestley-Taylor y redes neuronales artificiales en la estimación de la evapotranspiración de referencia. Ingeniería Investigación y Tecnología. v. XIV, n. 2, p. 163-176, 2013.

FENG, Y.; JIA, Y.; CUI, N.; ZHAO, L.; LI, C.; GONG, D. Calibration of Hargreaves model for reference evapotranspiration estimation in Sichuan basin of southwest China. Agricultural Water Management. v.181, p.1-9, 2017. https://doi.org/10.1016/j.agwat.2016.11.010

FERREIRA, L. B.; CUNHA, F. F.; DUARTE, A. B.; SEDIYAMA, G. C.; CECON, P. R. Métodos de calibração para a equação de Hargreaves-Samani. Ciência e Agrotecnologia, Viçosa, v. 42, n.1, p. 104$114,2018$.

HARGREAVES, G.H. Climate and irrigation requirements for Brazil. Logan, Utah State Universitey, 1976. 44 p.

HARGREAVES, G.H. Defining and using reference evapotranspiration. Journal of 
Irrigation and Drainage Engineering, v.120, n.6, p.1132-1139, 1994.

HARGREAVES, G. H.; SAMANI, Z. A. Reference crop evapotranspiration from temperature. Applied Engineering in Agriculture, v. 01, p. 96-99, 1985.

HEYDARI, M.M; HEYDARI, M. "Calibration of Hargreaves-Samani equation for estimating reference evapotranspiration in semiarid and arid regions". Archives of Agronomy and Soil Science, v. 60, p. 695-713, 2014. https://doi.org/10.1080/03650340.2013.80874

LACERDA, Z. C.; TURCO, J. E. P. Estimation methods of reference evapotranspiration (ETo) for Uberlândia MG. Engenharia Agrícola, v.35, n.1, p. 2738, 2015. http://dx.doi.org/10.1590/18094430-Eng.Agric.v35n1p27-38/2015

LIMA JUNIOR, J. C.; ARRAES, F. D. D.; OLIVEIRA, J. B.; NASCIMENTO, F. A. L.; MACÊDO, K. G. Parametrização da equação de Hargreaves e Samani para estimativa da evapotranspiração de referência no Estado do Ceará, Brasil. Revista Ciência Agronômica, v.47, n.3, p.447-454, 2016. https://doi.org/10.5935/1806-6690.20160054

MEHDIZADEH, S.; SAADATNEJADGHARAHASSANLOU, H.; BEHMANESH, J. "Calibration of HargreavesSamani and Priestley - Taylor equations in estimating reference evapotranspiration in the Northwest of Iran". Archives of Agronomy and Soil Science, v.63, p. 1-14, 2016. https://doi.org/10.1080/03650340.2016

MENDICINO, G.; SENATONE, A. Regionalization of the Hagreaves coefficient for assessment of distributed reference evapotranspiration in southern Italy. Journal of Irrigation and Drainage Engineering, North Carolina, v. 139, n. 5, p. 349-362, 2013. OLIVEIRA, P. J. D. de. Integridade dos dados meteorológicos obtidos por duas estações meteorológicas automatizadas. 2014. 44 f. Dissertação (Mestrado em Agronomia) - Faculdade de Ciências Agrárias e Veterinárias, Universidade Estadual Paulista, Jaboticabal, 2014. https://doi.org/10.15809/irriga.2019v24n2p35 2-366

OLIVEIRA, P. J. D. de; TURCO, J.E.P. Dois métodos de estimativa da evapotranspiração de referência e índices de estresse hídrico em cebola irrigada. Irriga: Brazilian Journal of Irrigation and Drainage. Botucatu-SP, v. 24, n.2, p. 352-366. 2019.

RAMOS, F.; OCCHIPINTI, A. G.; VILA NOVA, N. A.; REICHART, K.; MAGALHÃES, P. C.; CLEARY, R. W. Engenharia hidrológica. Rio de Janeiro: UFRJ, 1989. 404p.

RAZIEI, T.; PEREIRA, L.S. Estimation of ETo Hargreaves - Samani and FAO-PM temperature methods for a wide range of climates in Iran. Agricultural Water Management. v.121, p.1-18, 2013. https://doi.org/10.1016/j.agwat.2012.12.019

SILVA, J. R. L.; MONTENEGRO, A. A. A.; SANTOS, T. E. M.; SANTOS, E. S. Desempenho de diferentes métodos de estimativa da evapotranspiração de referência para Fernando de Noronha. Irriga, v.19, n.3, p. 390-404, 2014.

TRAJKOVIC, S. Hargreaves versus PenmanMonteith under humid conditions. Journal of Irrigation and Drainage Engineering. v.133, n.1, p. 38-42. 2007.

TURCO, J.E.P.; BARBOSA, J.C. Avaliação de duas estações meteorológicas automatizadas, para estimativa diária da evapotranspiração de referência obtida pelo método de Penman-Monteith. Irriga: Brazilian Journal of Irrigation and 
Turco J. E. P.

Drainage, Botucatu-SP, v.13, n.3, p.339-354. 2008.

TURCO, J.E.P.; CARLETO, N. Integridade de dados meteorológicos para uso em modelo hidrológico. Revista brasileira de agricultura irrigada - RBAI, Fortaleza-CE, v.11, n.8, p. 2084 - 2097, 2017.

TURCO, J. E. P.; PINOTTI, J. R.; PAVANI,
L. C.; FERNANDES, E. J. Técnica para determinação de erros em métodos de estimativa da evapotranspiração de referência. Engenharia Agrícola, Jaboticabal, v. 14, p. 31-37, 1994.

VUOLO, J.E. Fundamentos da teoria de erros. 2.ed. São Paulo: Edgard Blucher, 1996. 249p. 